\title{
Co-compostage de résidus solides de toilettes sèches mobiles en présence de déchets verts
}

\section{KOANDA A. ${ }^{a}$, BAYARD R. ${ }^{a}$, NAQUIN P. ${ }^{\mathrm{a}, \mathrm{b}}$, JEAN G. ${ }^{\mathrm{b}, \mathrm{c}}$, BÉVALOT F. ${ }^{\mathrm{d}}$, BOTTINELLI C. ${ }^{\mathrm{d}}$ et GOURDON R. ${ }^{\mathrm{a}}$}

a. Université de Lyon, INSA Lyon, LGCIE - Laboratoire de Génie Civil et d'Ingénierie Environnementale 20 avenue Albert Einstein, 6962I Villeurbanne cedex, France b. CEFREPADE, INSA Lyon, F-6962I Villeurbanne Cedex, France

c. Université Quisqueya, LAQUE - Laboratoire de Qualité de l'Eau et de l'Environnement BP 796, Port-au-Prince, Haïti. d. Laboratoire LAT LUMTOX, 7 I avenue Rockefeller, 69003 Lyon, France

\section{Auteur/s à qui la correspondance devrait être adressée : remy.bayard@insa-lyon.fr}

\section{RESUME}

Parmi les techniques de gestion et de traitement des excrétas solides et liquides humains, les toilettes à litière biomaîtrisée (TLB) ou toilettes sèches (TS) offrent une solution alternative aux systèmes utilisant l'eau. En fort développement dans les pays scandinaves, le principe des TS est de collecter les urines et les feces, mélangées à un absorbant solide. La filière TS présente l'avantage de permettre la valorisation directe après compostage, sans besoin de déshydratation, des excrétas humains en tant qu'amendement organique pour l'agriculture. Cependant il existe encore peu de retours d'expériences scientifiques permettant de déterminer les conditions optimales de traitement de ces résidus au cours d'un compostage, afin de se conformer aux exigences de qualité et d'innocuité des composts.

La présente étude sintéresse plus particulièrement aux toilettes sèches mobiles (TSM) et considère l'option d'une filière de cocompostage de résidus de TSM avec des déchets d'origine végétale. L'objectif expérimental était l'étude de leur biodégradabilité en conditions aérobies lors d'essais de compostage en pilotes de laboratoire avec ou sans ajout de déchets verts en différentes proportions. Réalisée sur des résidus de TSM collectés en France lors d'un festival de musique, l'étude a permis de mettre en évidence l'absence d'agents pathogènes dans les échantillons après 6 mois de stockage en cuves non aérées et leur bonne biodégradation en conditions de compostage en présence de déchets verts. La mise en évidence de plusieurs micropolluants médicamenteux (anti-inflammatoires non stéroïdiens, antalgiques, antibiotiques, hormones etc.) et stupéfiants (famille des opiacés, des cocaïniques, des amphétaminiques, et cannabinoïdes) a conduit à s'intéresser à leur devenir dans les essais de co-compostage. Les résultats obtenus après 3 semaines de compostage révèlent la biodégradation significative (plus de 75\%) de plus des 2/3 des micropolluants pharmaceutiques (MPP) analysés (diclofenac, Kekétoprofene, ciprofloxacine, paracétamol, propanolol, éconazole et ibuprofene) ainsi que pour les stupéfiants analysés tels que la morphine, l'amphétamine et la MDMA (MéthylèneDioxyMéthAmphétamine ou ecstasy). Cependant certaines molécules présentent des caractères de persistance dans les résidus étudiés ; c'est le cas de l'acide salicylique, de l'érythromycine, de la kétamine, de la norkétamine, du tramadol et de la MDA (méthylène dioxyamphétamine), un produit de dégradation de la MDMA.

\section{ABSTRACT}

Among techniques for human solid and liquid wastes management and treatment, Dry Toilets (DT) are often used as a possible solution for flush toilets issues. Highly developed in Scandinavian countries, the principle of DT is to collect separately urine and feces, mixed with a solid absorbent. DT technologies have an advantage to allow human wastes to be directly valorized, without dehydration after composting as a fertilizer for agriculture. However there are still few scientific researche to determine the optimum composting process conditions for these kinds of wastes, to comply with composts quality and safety.

This study is especially interested to Mobile Dry Toilets (MDT) and considers the option MDT wastes co-composting with vegetable wastes. This experimental aim is to study MDT wastes biodegradability under aerobic conditions, by considering their mixing with or without vegetable wastes in different proportions. Performed on MDT residues collected on electronic festival music, the samples' bio-physico and chemical characterization has highlighted the absence of pathogens after 6 months in storage tanks and showed the biodegradation ability of such residues in aerobic conditions mixed with vegetable wastes. Highlighting several pharmaceutical micropollutants (such as no-steroidal anti-inflammatory drugs, analgesics, antibiotics, hormones) and drugs (such as opiates, cocaine, amphetamines and cannabinoids compound families) has led to interest in their fate during co-composting process testing in laboratory pilots (25\%, 35\%, 50\% RTS).

An overview of the obtained results after 3 weeks composting process shows a good removal for more than $70 \%$ of pharmaceutical micropollutants analyzed (diclofenac, ketoprofen, ciprofloxacin, paracetamol, Propranolol, econazole and ibuprofen.) and for drugs compounds as amphetamine and MDMA or ectasy a narcotic. However, some molecules have characteristics of persistence in the samples studied; it is the case of salicylic acid and erythromycin pharmaceutical micropollutants, ketamine, norketamine, tramadol and MDA.

KEYWORDS :Composting, dry toilet residues, mobile dry toilets, green waste, pharmaceutical micropollutants, pathogenic agents, drugs, respiration, maturity, indicators of stability. 


\section{Co-compostage de résidus solides de toilettes sèches mobiles en présence de déchets verts}

KOANDA A., BAYARD R., NAQUIN P., JEAN G., BÉVALOT F., BOTTINELLI C. et GOURDON R

\section{INTRODUCTION}

Malgré le développement du traitement collectif ou individuel des eaux usées domestiques, la dispersion des germes pathogènes et composés pharmaceutiques dans l'environnement est avérée par de nombreux travaux de recherche (Daughton et Ternes, 1999 ; Jjemba, 2006 ; Jones et al., 2005 ; Kümmerer, 200 I ; Zuccato et al., 2000). Les conséquences de la dispersion des composés pharmaceutiques sont encore mal connues, mais leur implication dans certaines maladies comme le cancer, les maladies de Parkinson et d'Alzheimer est suspectée (Winker, 2009).

Dans un contexte de fort développement de la consommation de principes actifs à usage médical, la difficulté et le coût du traitement des micropolluants en stations d'épuration (Jones et al., 2005) justifient de s'intéresser au développement de filières alternatives. Parmi elles, les toilettes sèches (TS), appelées également toilettes à litière biomaîtrisée (TLB) sont une alternative en fort développement dans certains pays notamment européens (http://www.drytoilet.org/dt2012/ ). Les TS présenteraient l'avantage par rapport aux filières traditionnelles d'assainissement liquide, de réduire les risques de dispersion des micropolluants et germes pathogènes, et de permettre la valorisation directe, sans besoin de déshydratation après compostage, des excrétas humains en tant que fertilisants pour l'agriculture (Pynnönen \& Tuhkanen, 2012) Cependant, malgré les avantages pressentis, la question se pose sur le choix de la filière de traitement-valorisation et les risques associés.

Le compostage est perçu comme une option adaptée de traitement, mais il existe encore peu de retours d'expériences scientifiques permettant de le démontrer, et de déterminer les conditions optimales de compostage des résidus solides de TS. Cette étude vise à évaluer si le traitement biologique par co-compostage des résidus solides de toilettes sèches (RTS) issus de festivals de musique, peut être envisagé. II s'agit plus précisément d'étudier d'une part leur biodégradabilité en conditions aérobies avec ou sans ajout de déchets verts (DV) en différentes proportions, et d'autre part, le devenir de micropolluants pharmaceutiques et stupéfiants susceptibles d'être présents.

Des essais de compostage sur pilotes en laboratoire (conditions contrôlées d'apport en oxygène) ont permis de comparer l'activité biologique (température, consommation de $\mathrm{O}_{2}$, production de $\mathrm{CO}_{2}$ et bilans matières) du mélange RTS et déchets verts en fonction du ratio RTS/DV. De même, une évaluation de la biodégradation des micropolluants initialement présents dans les résidus de TSM est également mise en évidence au cours de ces essais. Le suivi des agents pathogènes après compostage n'est pas l'objet de cette étude, compte tenu de leur faible concentration lors de la caractérisation initiale des RTS.

\section{Matériel et méthodes}

\section{I. Origine, collecte, stockage et conditions de préparation des échantillons}

Les résidus de toilettes sèches (RTS) mobiles ont été collectés lors du festival de musique électronique en France (novembre 2012). Après collecte en containers étanches de $1 \mathrm{~m}^{3}$ $\left(\left|m^{*}\right| m^{*} \mid m\right)$ non couverts sur le site du festival, les résidus ont été stockés à l'extérieur pendant 6 mois. Ces résidus sont essentiellement constitués d'excréments solides et liquides, et d'un mélange de différentes sciures et copeaux de bois issus de scieries. Le prélèvement des échantillons a été effectué en avril 2013 sur un choix aléatoire de deux cuves de stockage parmi les 12 cuves disponibles, dont le contenu a été soigneusement brassé afin de collecter un échantillon d'une centaine de kilogrammes représentatif du lot initial. Après la collecte d'un échantillon d'environ $200 \mathrm{~kg}$ de matière humide, les RTS ont été aliquotés dans les seaux de $20 \mathrm{~L}$ et stockés en chambre froide $\left(5 \pm 2^{\circ} \mathrm{C}\right)$, avant réalisation des essais de caractérisation et de compostage.

Les déchets verts (DV), broyés mécaniquement (> $20 \mathrm{~mm}$ ) mais non compostés, proviennent d'une plateforme de compostage de la Région Rhône-Alpes, spécialisée dans le traitement de déchets organiques issus de l'entretien d'espaces verts de collectivités et de particuliers. Après collecte d'environ $300 \mathrm{~kg}$ de masse humide, $50 \mathrm{~kg}$ du lot ont été broyés dans un broyeur Blik ${ }^{\circledR}$ M420 et criblés à $<10$ mm. Les échantillons ont été stockés en chambre froide $\left(5 \pm 2^{\circ} \mathrm{C}\right)$ avant réalisation des essais de caractérisation et de compostage.

\subsection{Caractérisation bio-physico-chimique}

Matière sèche et matière organique : la détermination des teneurs pondérales en eau et matières des RTS et déchets 
verts a été réalisée sur échantillons initiaux et obtenus après compostage. Le taux de matière organique sur échantillons solides secs et broyés est mesuré selon la méthode de la perte au feu (matière volatile : MV) par calcination à $550^{\circ} \mathrm{C}$ dans un four à moufles pendant $4 \mathrm{~h}$. Les résultats sont exprimés en pourcentage de matière sèche $\left(\%_{M S}\right)$. La seconde méthode de quantification de la matière organique totale est la demande chimique en oxygène (DCO) déterminée sur échantillon solide par oxydation sulfochromique en présence de bichromate de potassium. Le protocole développé au LGCIE se réfère à la norme internationale ISO EN NF I 4235 (1998). Les analyses du carbone total $(\mathrm{C})$, de l'azote total $(\mathrm{N})$, de l'azote organique $\left(\mathrm{N}-\mathrm{N}_{\text {org }}\right)$ et de l'azote ammoniacal $\left(\mathrm{N}-\mathrm{NH}_{3}\right)$ ont été effectuées par le Laboratoire Départemental d'Analyses et de Recherche (LDAR) suivant les normes ISO spécifiques en vigueur pour l'analyse de compost de boues de station d'épuration AFNOR NF U 44-095 (2002). Enfin, la capacité de rétention en eau (CRE) des échantillons a été déterminée sur les deux matrices selon une méthode au Büchner développée au LGCIE. C'est la quantité maximale d'eau capillaire retenue par le milieu. Elle consiste à introduire dans un flacon muni d'un couvercle environ $50 \mathrm{~g}$ de masse humide de déchet et recouvrir d'eau déminéralisée (environ $100 \mathrm{~mL}$ ) pendant I h, et laisser écouler l'eau sous une dépression de 100000 Pa pendant 30 min.

Taux de conversion de matière organique et cinétique de biodégradation : les taux de conversion $\tau$ la matière organique dans les quatre essais à 21 jours d'incubation ont été déterminés sur la base de la production de $\mathrm{CO}_{2}$, en considérant l'hypothèse que la composition stœchiométrique de la matière organique présente dans les deux matrices (RTS, DV) s'apparente à la stœchiométrie de la molécule de glucose $\left(\mathrm{C}_{6} \mathrm{H}_{12} \mathrm{O}_{6}\right)$ et des équations suivantes.

Réaction stœchiométrique (cas du glucose)

$$
\mathrm{C}_{6} \mathrm{H}_{12} \mathrm{O}_{6}+6 \mathrm{O}_{2} \text { à } 6 \mathrm{CO}_{2}+6 \mathrm{H}_{2} \mathrm{O}(1)
$$

Taux de conversion :

$\mathrm{T}\left(\%_{\mathrm{MV}}\right)=Q_{\mathrm{CO} 2} * \frac{\text { MM subst.org}}{M M \text { co2 }} * \frac{1}{1000} * \mathbf{1 0 0} * \frac{100}{M V_{\% M S}}(2)$

Avec $\tau$ exprimé en pourcentage de matière organique converti en $\mathrm{CO}_{2} ; \mathrm{Q}_{\mathrm{CO} 2}$, la production cumulée de $\mathrm{CO}_{2}$ et $\mathrm{MV}_{\% \mathrm{MS}}$ le taux de matière volatile dans la matière sèche présente dans le réacteur.

La constante cinétique globale de biodégradation de la matière organique a été déterminée selon le modèle cinétique de 1 er ordre:

Constante de cinétique de premier ordre:

$Q_{\mathrm{CO} 2}=Q_{\mathrm{CO} 2 \operatorname{Max}} \times\left(\mathbf{1}-e^{(-k \times t)}\right)(3)$

(Constante de cinétique exprimée par rapport à la quantité de MS dans le réacteur).

\section{Analyse microbiologique de l'échantillon de RTS} l'analyse des agents pathogènes cités dans la norme compost de boues AFNOR NFU-44095 a été réalisée par le
Laboratoire Départemental d'Analyses et de Recherche de l'Aisne (LDAR) selon les procédures standards sur l'échantilIon initial de RTS : Escherichia coli (NF ISO 16649-2), Clostridium perfringens (NF EN ISO 7937), entérocoques (NF EN ISO 7899-I), Salmonella (NF EN ISO 6579), et œufs d'Helminthes viables (Méthode de Baërmann).

Evaluation de la capacité d'auto-échauffement (CAE) : le principe de cette étude consiste à rechercher le degré de décomposition de la matière organique biodégradable dans un compost ou un déchet organique en déterminant la capacité d'échauffement naturel $\left(\Delta T=T_{\text {Max }}-T_{\text {amb }}\right)$, et de distinguer les matrices selon une classe de biodégradabilité selon le protocole développé par Brinton et al. (1995). Elle est réalisée en vase DEWAR. Quatre essais ont été effectués (25, 35,50 et $100 \%_{M H}$ de RTS) comparativement à l'essai matrice témoin $100 \%_{M H}$ déchet organique.

\section{Evaluation de l'activité respiratoire en conditions} statiques d'aération (ARS) : basée sur la procédure décrite dans la Ligne Directrice 304A de l'OCDE (1981) pour la mesure de l'activité respiratoire d'échantillons de sols, ce test de respirométrie permet de déterminer la consommation en oxygène d'un déchet solide, sous des conditions de renouvellement ponctuel de l'air. Pour cela, $10 \mathrm{~g}$ de compost humide (RTS et DV) à leur capacité de rétention en eau et proportions respectives ont été placés dans un jarre de 900 $\mathrm{mL}$ OxiTop ${ }^{\circledR}$ contenant un bécher de $30 \mathrm{~mL}$ de soude à 2,5 $\mathrm{N}$ pour piéger le $\mathrm{CO}_{2}$. Le compost est incubé pendant 10 jours à $20^{\circ} \mathrm{C}$. Le système OxiTop ${ }^{\circledR} \mathrm{OC} 110$ mesure en continu la dépression dans le flacon et permet de calculer une consommation d' $\mathrm{O}_{2}$. Les résultats sont exprimés en $\mathrm{mg} \mathrm{d}^{\prime} \mathrm{O}_{2}$ consommé par $g_{M S^{\prime}}$ Quatre essais ont été effectués $(25,35$, 50 et $100 \% \mathrm{MH}$ de RTS) et $10 \mathrm{~g}_{\mathrm{MH}}$ de matrice finale ont été utilisés. Les proportions de mélange entre les deux matrices tiennent compte de l'humidité $80 \%$ pour les RTS et $66 \%$ pour les déchets organiques à leur capacité de rétention en eau.

\subsection{Dosage des micropolluants}

Analyse des micropolluants pharmaceutiques : le dépistage ciblé de résidus médicamenteux a été réalisé par le Service Central d'Analyse de l'Institut des Sciences Analytiques de Lyon, France. Le choix des molécules cibles dans cette analyse par screening a été effectué en tenant compte des classes thérapeutiques des médicaments les plus prescrits en France. Nous avons également croisé ces informations avec les données de la littérature en prenant en considération leurs critères d'occurrence dans les effluents de stations d'épuration urbaines, et aussi, en fonction de leur persistance dans l'environnement, 12 micropolluants pharmaceutiques (MPP) ont été recherchés dans les RTS solides initiaux et les matrices solides après incubation dans les composteurs de laboratoire, classés en cinq familles thérapeutiques : antalgiques (paracétamol), anti-inflammatoires non stérö̈diens (acide salicylique, 
diclofénac, ibuprofène, kétoprofène), bêtabloquants (aténolol, propanolol), hormones (éthynil œstradiol), antibiotiques (ciprofloxacine, éconazole, érythromycine), et antidépresseurs (carbamazépine). Les MPP sont extraits sur $2 g_{\text {MS }}$ au solvant (extracteur automatique ASE DIONEX ${ }^{\circledR}$ ), avec purification en phase solide (SPE) pour la ciprofloxacine sur cartouche Oasis ${ }^{\circledR} H L B$ et SPE dispersive (QuEChERS ${ }^{\circledR}$ ) pour les autres molécules, et analysés par LC/MS-MS en mode d'ionisation electrospray positif et négatif. Sur matrices liquides, les MPP sont extraits et purifiés par SPE sur cartouche Oasis ${ }^{\circledR} H L B$, puis analysés également par LC/MSMS en mode d'ionisation electrospray positif et négatif.

Autres analyses : Des analyses complémentaires ont été réalisées au sein du laboratoire d'analyses toxicologiques LAT LUMTOX, Lyon, France. Un dépistage large (screening) de xénobiotiques (stupéfiants et molécules médicamenteuses) a été effectué dans les échantillons solides secs et humides des résidus de toilettes sèches. Ce screening a consisté en une extraction liquide-liquide (LLE) suivie d'une acétylation. Les extraits ont été analysés par chromatographie en phase gazeuse couplée à un détecteur de type spectromètre de masse (GCMS) (GC 6890, MSD 5973, Agilent). Des analyses spécifiques ont été réalisées sur les 4 principales familles de stupéfiants: les opiacés (morphine, 6-monoacétylmorphine ou 6MAM), les cocaïniques (benzoylecgonine (BZE) et ecgonine methylester (EME) métabolites de la cocaïne), les amphétaminiques (amphétamine, méthamphétamine, ecstasy ou MDMA, et MDA dérivé de l'ecstasy), ainsi que les cannabinoïdes (tétra-hydrocannabinol THC et ses deux métabolites, le II-OH-THC, et le $\mathrm{THC}-\mathrm{COOH}$ ). Toutes les analyses spécifiques mettent en œuvre des extractions de type LLE. L'extraction des opiacés et des cocaïniques est commune à $\mathrm{pH}$ basique. Les extraits sont ensuite silylés. Les amphétaminiques sont extraits par acétate d'éthyle (AE) en milieu basique puis analysés sous forme de dérivés trifluoroacétylés. Les extraits de ces 3 familles de stupéfiants ont été analysés par GC-MS. Les cannabinoïdes ont été extraits par un mélange hexane/AE ( $9: 1 \mathrm{v} / \mathrm{v})$ puis méthylés. Leur analyse a été effectuée par chromatographie en phase gazeuse couplée à un spectromètre de masse en tandem (GC-
MS/MS) en mode Multiple Reaction Monitoring (MRM).

Le taux d'abattement des micropolluants est calculé sur la base du rapport de la différence entre la quantité initiale et finale de micropolluants avant et après incubation, et la quantité initiale avant incubation. Les quantités de micropolluants dans les matrices d'essais étudiées sont calculées sur la base de la masse sèche de RTS contenue dans la matrice étudiée multipliée par la concentration mesurée. Pour illustration, les quantités initiales et finales de MPP dans l'essai 25\% de RTS se calculent comme suit

$\mathrm{Qi}_{25 \% \mathrm{RTS}}=$ Masse sèche initiale RTS dans la matrice * Concentration initiale MPP dans la matrice

$\mathrm{Qf}_{25 \% \mathrm{RTS}}=$ Masse sèche RTS dans la matrice $(\mathrm{I}-\boldsymbol{\tau}) *$ Concentration finale MPP dans la matrice

Cette dernière valeur de masse sèche de la matrice finale se calcule en retranchant la masse perdue au cours de la biodégradation.

$\mathrm{Ab}=\frac{\text { Quantité initiale } M P P-Q \text { uantité finale } M P P}{\text { Quantité initiale } M P P}$

\subsection{Essais de compostage en pilotes}

Les tests en pilotes de compostage ont pour objectif d'évaluer le comportement des déchets (DV, RTS et MIX), mais dans des conditions régulées d'aération (incubation de trois semaines et sur des quantités de matière de l'ordre de 50 kg ${ }_{\mathrm{MH}}$ ), situation permettant de simuler des conditions proches d'un dispositif industriel de compostage en conditions d'aération contrôlée (cf. Figure I). Brièvement, l'atmosphère du réacteur est recirculée en continu au travers de la colonne de déchets de bas en haut. Lorsque la teneur en $\mathrm{O}_{2}$ est inférieure à $15 \%$, de l'air frais est automatiquement réinjecté pour maintenir la concentration de consigne. La température n'est pas régulée, la paroi isolante permettant de limiter les pertes thermiques. La température, et les teneurs en $\mathrm{O}_{2}$ et $\mathrm{CO}_{2}$ ont été suivies au cours des quatre essais décrits dans le Tableau I. La siccité initiale pour les RTS est de $80 \%$ en humidité, et celle des déchets verts bruts de $61 \%$. Le débit de réinjection d'air est de 16,5 L/mn soit environ $50 \mathrm{~L}^{\mathrm{L}} \mathrm{h}^{-1} \cdot \mathrm{kg}^{-1}$ MS

Tableau I. Conditions opératoires des essais de compostage en pilote de laboratoire.

\begin{tabular}{|c|c|c|c|c|c|c|c|c|}
\hline Essai & $\begin{array}{l}\text { Déchet } \\
\text { Organique } \\
\text { (\% MH) }\end{array}$ & $\begin{array}{c}\text { RTS } \\
\text { (\% MH) }\end{array}$ & $\begin{array}{l}\text { Siccité } \\
(\% \text { MH) }\end{array}$ & $\begin{array}{c}\text { Masse } \\
\text { humide } \\
(\mathrm{kgMH})\end{array}$ & $\begin{array}{c}\text { Masse sèche } \\
(\mathrm{kgMH}) \\
\text { DV et RTS }\end{array}$ & $\begin{array}{l}\text { Matière Orga- } \\
\text { nique (kgMH) } \\
\text { DV et RTS }\end{array}$ & Densité & Hauteur (m) \\
\hline EI & 100 & - & $\begin{array}{c}\text { Initiale } \\
(61,3 \pm 0,9 \%)\end{array}$ & 51,1 & 19,8 & 14,9 & 0,415 & 0,65 \\
\hline E2 & 75 & 25 & $\begin{array}{c}\text { Initiale } \\
(68,3 \pm 0,6 \%)\end{array}$ & 51,1 & $\begin{array}{c}17,4 \\
\text { DV : | } 4,8(85 \%) \\
\text { RTS : } 2,5(\mid 5 \%)\end{array}$ & $\begin{array}{c}13,49 \\
\text { DV: I I, } 11 \text { (83\%) } \\
\text { RTS : } 2,38(17 \%)\end{array}$ & 0,429 & 0,63 \\
\hline E3 & 65 & 35 & $\begin{array}{c}\text { Initiale } \\
(70,6 \pm 0,9 \%)\end{array}$ & 51,2 & $\begin{array}{c}16,4 \\
\text { DV : } 12,9(78,7 \%) \\
\text { RTS : } 3,5(21,3 \%)\end{array}$ & $\begin{array}{c}13,00 \\
\text { DV: } 9,7(75 \%) \\
\text { RTS : } 3,3(25 \%)\end{array}$ & 0,494 & 0,55 \\
\hline E4 & 50 & 50 & $\begin{array}{c}\text { Initiale } \\
(73,4 \pm 0,5 \%)\end{array}$ & 51,2 & $\begin{array}{c}15 \\
\text { DV }: 9,9(66 \%) \\
\text { RTS : } 5,1(34 \%)\end{array}$ & $\begin{array}{c}12,3 \\
\text { DV: } 7,4(60 \%) \\
\text { RTS }: 4,9(40 \%)\end{array}$ & 0,513 & 0,53 \\
\hline
\end{tabular}




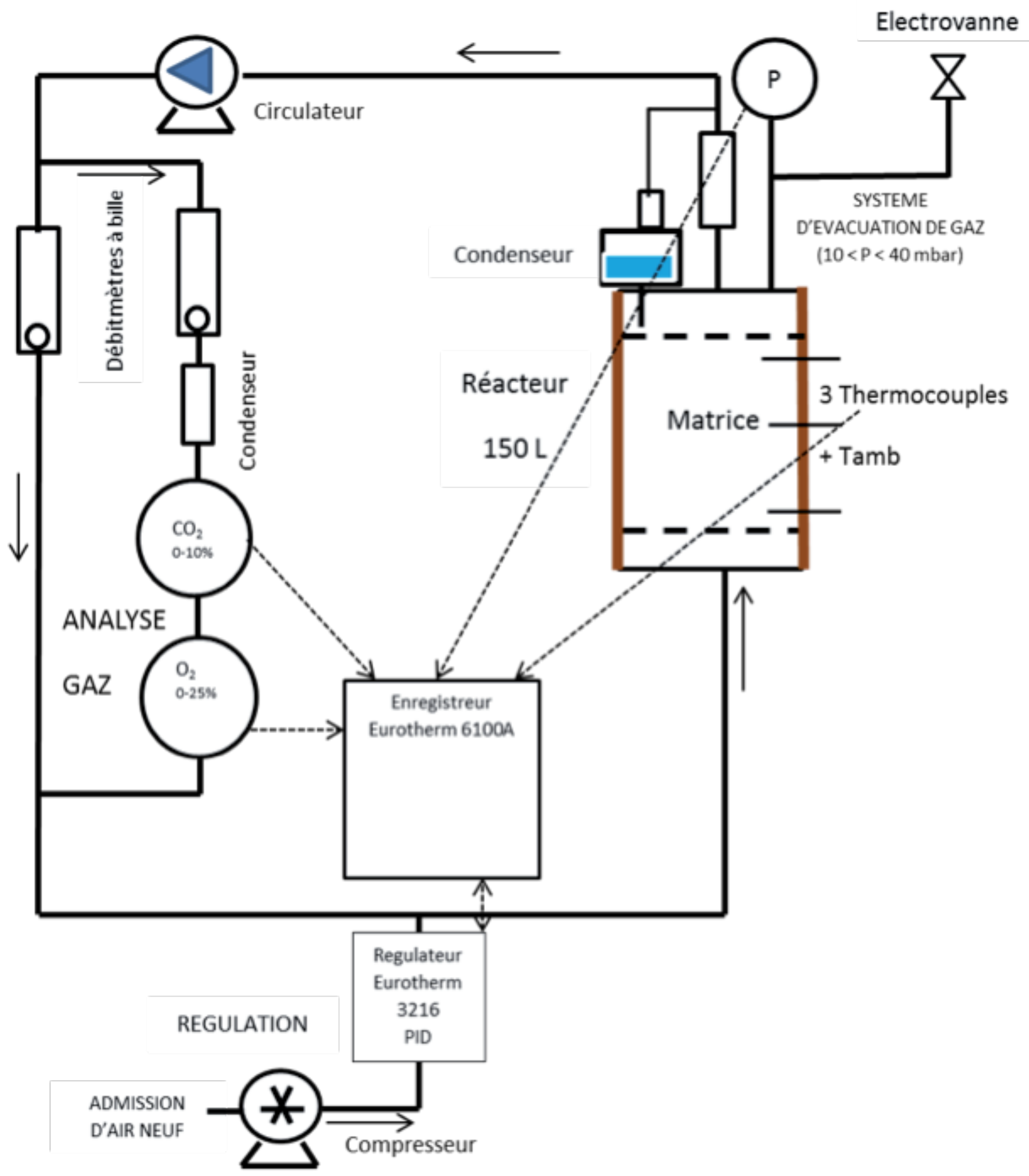

Figure I : Schéma du dispositif pilote de compostage. 


\section{Résultats et discussion}

\section{I. Caractéristiques des matrices initiales}

Analyse globale : Les résultats d'analyse globale des échantillons initiaux de résidus de toilettes sèches (RTS) et de déchets verts (DV) sont présentés dans le Tableau 2. Les RTS se caractérisent par une humidité élevée $\left(80 \%_{\mathrm{MH}}\right)$, proche de leur capacité de rétention en eau et nettement supérieure aux valeurs optimales considérées pour le compostage, généralement comprises entre 50 et 60\% (Mustin, 1987). Les DV collectés pour les essais de co-compostage présentent un taux d'humidité d'environ $60 \%{ }_{\mathrm{MH}}$, soit une valeur inférieure à la CRE.

Les TS et les DV présentent tous deux des ratios C/N favorables à leur compostage (37 et 28 , respectivement ${ }^{(I)}$, cf. Tableau 2). Les analyses indiquent également que dans les deux matrices l'azote se trouve à plus de $80 \%$ sous forme organique (principalement uréique). L'azote sous forme ammoniacale $\left(\mathrm{NH}_{3}\right)$ dans ces matrices représente seulement 10\% de l'azote total.

Par ailleurs, en termes de réactivité biologique, les résultats montrent une meilleure biodégradabilité des DV en comparaison avec les RTS. Cette différence de réactivité est particulièrement manifeste au travers des tests de capacité d'auto-échauffement ( $\mathrm{CAE}$ ), où l'auto-échauffement des déchets verts est de 35,4 \pm I,7, et celui des RTS seulement de 2,3 $\pm 0,4$. Toutefois, cette faible réactivité des RTS est due à leur forte teneur initiale en eau (80\%), proche de la capacité de rétention du déchet, et vraisemblablement défavorable à l'aération passive du résidu au cours de l'essai CAE ainsi qu'à son auto-échauffement. Les résultats du test d'activité respiratoire en conditions statiques (ARS) confirment cette hypothèse. En effet, la consommation d'oxygène après 14 jours d'incubation des RTS est plus faible que celle des DV (respectivement environ 100 et 160 g. $\mathrm{kg}^{-1}$ MS $)$, sans toutefois reproduire l'ampleur de l'écart observé dans la mesure de capacité d'auto-échauffement. Ces activités respiratoires correspondent à 7\% et 15\% de bioconversion de la DCO initialement présente dans les RTS et DV. En conclusion, ces premières caractérisations des matrices tendent à mettre en évidence que les RTS, matrices très humides et potentiellement biodégradables en conditions aérobies, ne sont pas compostables telles quelles. La réduction de la teneur en humidité et le co-compostage en présence d'un co-substrat plus sec et plus structurant paraissent donc nécessaires.

Recherche de germes pathogènes dans les RTS : les concentrations en germes pathogènes sont nettement inférieures aux seuils de la norme de valorisation compost (cf. Tableau 3). Ces faibles concentrations en germes pathogènes dans les RTS pourraient s'expliquer par la durée et les conditions de la période de stockage (6 mois environ) entre leur génération et la campagne de prélèvement. De même, il est probable que la température (entre 20 et $30^{\circ} \mathrm{C}$ ) et les conditions alcalines $(\mathrm{pH}=8,9)$ pendant cette phase de stockage soient également des facteurs défavorables à la survie des germes pathogènes (Baumeyer, 2003). La biodégradation de l'urée en ammoniac pourrait expliquer l'installation des conditions alcalines dans les résidus humides des toilettes sèches, contribuant ainsi à l'abattement des germes pathogènes.

Présence de micropolluants pharmaceutiques et stupéfiants : l'analyse par screening de la matrice initiale de RTS a révélé la présence de 9 micropolluants pharmaceutiques (MPP), 6 stupéfiants et 3 xénobiotiques (et métabolites). Les résultats sont résumés dans les Tableau 4A et 4.B.

(I) La présence d'une forte quantité de sciure et de copeau de bois dans le RTS traduit une teneur plus élevée de carbone dans ces derniers que dans les DV, justifiant ainsi leur C/N plus important.

Tableau 2. Caractéristiques globales des matrices initiales.

\begin{tabular}{|c|c|c|c|c|c|c|c|c|c|c|c|c|}
\hline Matrices & $\begin{array}{l}\text { Humidité } \\
\text { (\%MH) }\end{array}$ & pH & $\begin{array}{c}\text { MS } \\
(\% / M H)\end{array}$ & $\begin{array}{l}\text { CRE } \\
\text { (\%MH) }\end{array}$ & $\begin{array}{c}\text { MV } \\
(\% \text { MS) }\end{array}$ & $\begin{array}{c}\text { DCO } \\
(g / \mathrm{kg} M S)\end{array}$ & $\begin{array}{c}\text { N-NT } \\
(\mathrm{g} / \mathrm{kgMS}\end{array}$ & $\begin{array}{c}\mathrm{N}-N H_{3} \\
(\mathrm{~g} / \mathrm{kgMS})\end{array}$ & $\begin{array}{l}\mathrm{N}-\mathrm{N}_{\text {org }} \\
(\mathrm{g} / \mathrm{kgMS}\end{array}$ & $C^{*} / N$ & $\begin{array}{c}\text { CAE IO } \\
\Delta \mathrm{O}\left({ }^{\circ} \mathrm{C}\right)\end{array}$ & $\begin{array}{c}\text { ARSI4 } \\
\left(\mathrm{mgO}_{2} / \mathrm{g}_{\mathrm{HS}}\right)\end{array}$ \\
\hline RTS Solides & 80,1 & $8,9 \pm 0,1$ & $19,9 \pm 0,2$ & $80,0 \pm 0,5$ & $95,9 \pm 0,4$ & $1460 \pm 144$ & $1 \mathrm{II}, 7$ & 1,5 & 9,8 & 37,3 & $2,3 \pm 0,4$ & $102 \pm 7,0$ \\
\hline Déchets verts & 61,3 & $7,9 \pm 0,3$ & $38,7 \pm 0,9$ & $66,0 \pm 1,0$ & $75,1 \pm 3,6$ & $1070 \pm 20$ & 12,3 & $<1,0$ & 12,1 & 28,2 & $35,4 \pm 1,7$ & $160 \pm 2$ \\
\hline
\end{tabular}

* C déterminé par calcination.

Tableau 3. Analyse des agents pathogènes dans le résidu solide de TSM collecté

\begin{tabular}{|c|c|c|c|c|c|}
\hline Essais & E. coli/g & Clostridium perfringens/g & Entérocoques intestinaux (npp) & Salmonella $/ 25 g$ & Fufs helminthes /1.5 g \\
\hline RTS Solides & $<100$ & $<400$ & 2392 & Absence & Absence \\
\hline $\begin{array}{l}\text { Seuil de valorisation compost selon } \\
\text { la norme AFNOR NF U } 44-095\end{array}$ & $10^{4} / g$ M.B & $10^{2}-10^{3} / g \mathrm{MB}$ & $10^{5} / g$ M.B & $\begin{array}{l}\text { Absence dans I g de Matière } \\
\text { brute }\end{array}$ & $\begin{array}{c}\text { Absence dans I g de Matière } \\
\text { brute }\end{array}$ \\
\hline
\end{tabular}


Tableau 4. Dépistage ciblé de résidus médicamenteux (A) et stupéfiants (B) dans le résidu solide de TSM collecté.

\begin{tabular}{|c|c|c|}
\hline Classe thérapeutiques & Molécule & $\begin{array}{l}\text { Concentration } \\
\left(\mathrm{ng} / \mathrm{g}_{\mathrm{gS}}\right)\end{array}$ \\
\hline Antalgiques & Paracétamol & 71 \\
\hline \multirow{4}{*}{$\begin{array}{l}\text { anti-inflammatoire non } \\
\text { stéroïdiens }\end{array}$} & Ac. Salicylique & 568 \\
\hline & Diclofenac & 17 \\
\hline & lbuprofène & 716 \\
\hline & Kétoprofène & 200 \\
\hline \multirow{2}{*}{ Bêtabloquants } & Aténolol & ND \\
\hline & Propanolol & 55 \\
\hline Hormones & Ethynil œestradiol & ND \\
\hline \multirow{3}{*}{ Antibiotiques } & Ciprofloxacine & 502 \\
\hline & Econazole & 87 \\
\hline & Erythromycine & 60 \\
\hline Antidépresseurs & Carbamazépine & ND \\
\hline
\end{tabular}

(ND) non détecté (NQ) non quantifié

B

\begin{tabular}{|c|c|c|c|}
\hline $\begin{array}{l}\text { Classe de } \\
\text { stupéfiants }\end{array}$ & Molécule & $\begin{array}{l}\text { Concentration dans } \\
\text { RTS après séchage } \\
\left(\mathrm{ng} / \mathrm{g}_{\mathrm{MS}}\right)\end{array}$ & $\begin{array}{l}\text { Concentration dans } \\
\text { RTS humides } \\
\left(\mathrm{ng} / \mathrm{g}_{\mathrm{HS}}\right)\end{array}$ \\
\hline $\begin{array}{l}\text { Molécules médicamen- } \\
\text { teuses détournées de } \\
\text { leur usage }\end{array}$ & $\begin{array}{l}\text { Kétamine } \\
\text { Norkétamine } \\
\text { Tramadol }\end{array}$ & $\begin{array}{l}+ \\
+ \\
+\end{array}$ & $\begin{array}{l}+ \\
\text { ND } \\
+\end{array}$ \\
\hline \multirow{4}{*}{ Opiacés et cocainniques } & Morphine & 3.62 & 3.82 \\
\hline & GMAM & ND & ND \\
\hline & BZE & ND & ND \\
\hline & EME & ND & ND \\
\hline \multirow{4}{*}{ Amphétaminiques } & Amphétamine & 160 & 90 \\
\hline & Méthamphétamine & ND & ND \\
\hline & MDMA (Ecstasy) & 13000 & 7090 \\
\hline & MDA & 1160 & 410 \\
\hline \multirow{3}{*}{ Cannabinoïdes } & THC & ND & ND \\
\hline & II-OH-THC & ND & 47 \\
\hline & ТНС-COOH & ND & 117 \\
\hline
\end{tabular}

6MAM : 6-monoacétylmorphine (marqueur de l'héroine) ;

BZE : Benzoylecgonine (métabolite de la cocaine) ;

EME : Ecgonine MethylEster (métabolite de la cocaine) ;

MDMA : 3,4-méthylène-dioxy-methamphétamine ;

MDA : 3,4 méthylène-dioxy-amphétamine (dérivé et métabolite de la MDMA) ;

THC : tétra-hydro-cannabinol ;

| I-OH-THC : | I-hydroxy-tétrahydrocannabinol (métabolite duTHC)

$\mathrm{THC}-\mathrm{COOH}$ : carboxy-tétra-cannabinol (métabolite duTHC)

ND : Non Détecté.
Parmi les MPP recherchés, trois molécules n'ont pas été détectées dans les RTS initiaux (cf. Tableau 4.A). C'est le cas de l'éthynil œstradiol (hormone), la carbamazépine (antidépresseur) et l'aténolol (bêtabloquant), reflétant ainsi soit leur absence initialement dans les RTS ou encore leur biodégradation au cours des 6 mois de stockage. Quatre anti-inflammatoires sont mis en évidence dans l'échantillon de RTS, l'aspirine, l'ibuprofène, le kétoprofène et le diclofénac. La présence des trois antibiotiques ciblés est également confirmée, en particulier celle de la ciprofloxacine, antibiotique à large spectre, ainsi que d'un composé antalgique, le paracétamol, et d'un bêtabloquant, le propanolol. Pour les composés qui ont pu être détectés, les concentrations sont plutôt élevées (jusqu'à 750 $\mathrm{ng} \cdot \mathrm{g}^{-1}$ MS $)$, si on les compare à des valeurs obtenues dans des boues de station d'épuration (Soulier, 20 I I). D'après l'auteur, les valeurs de concentrations des substances pharmaceutiques dans les boues sont de l'ordre de la centaine de ng.g.' ${ }^{-1}$ ms les concentrations totales moyennes dans les effluents en entrée de STEP sont majoritairement inférieures au $\mu \mathrm{g} \cdot \mathrm{L}^{-1}$, sauf pour certaines substances largement utilisées comme le paracétamol ( $\left.80 \mu g \cdot \mathrm{L}^{-1}\right)$, l'aspirine ( $\left.30 \mu \mathrm{g} \cdot \mathrm{L}^{-1}\right)$, la théophylline ( $\left.4 \mu g . L^{-1}\right)$, l'ibuprofène ( I I $\left.\mu g . L^{-1}\right)$ et la caféine $\left(4 \mu g \cdot L^{-1}\right)$.

Le screening toxicologique a permis d'identifier la kétamine et un de ses métabolites, la norkétamine, ainsi que le tramadol, des molécules médicamenteuses détournées de leur utilisation dans un cadre de toxicomanie (la kétamine est une molécule utilisée notamment à des fins récréatives comme stupéfiant, le tramadol est un analgésique de la famille des opiacés). Les analyses spécifiques des stupéfiants conduisent cependant à la détection de morphine, d'amphétaminiques et de cannabinoïdes (cf. Tableau 4.B). Aucun produit cocaïnique n'a été mis en évidence avec ou sans séchage de l'échantillon avant extraction, résultats qui semblent indiquer leur absence dans les RTS, mais qui pourrait être liée à leur biodégradation après 6 mois de stockage. La présence de morphine en faible quantité peut indiquer la consommation d'héroïne. L'absence du marqueur 6MAM n'est pas significative car c'est une molécule peu stable. La détection des composés amphétaminiques est plus représentative dans les RTS solides secs que dans les RTS solides humides. Par ailleurs, les composés cannabinoïdes sont mieux détectés dans les RTS humides que dans les RTS secs. Soulignons également que les teneurs sont de l'ordre du $\mu g . g^{-1}$ à quelques dizaines de $\mu g . g^{-1}$ pour la molécule d'ecstasy et ses métabolites, soit des valeurs très faibles. Toutefois, les techniques d'extraction utilisées le sont sur des matrices type tissus humains. Leur validation sur matrices type biomasses lignocellulosiques parait nécessaire pour confirmer les résultats obtenus. Pour la suite, nous tiendrons compte des données de valeurs obtenues sur les matrices sèches pour l'interprétation de nos résultats d'abattement des molécules dans les différents essais de co-compostage. 
Figure 2 : Comparaison des cinétiques d'activité au cours du cycle de compostage (fermentation chaude de $2 \mathrm{I}$ jours) selon la proportion massique déchets verts / résidus de toilette sèche mobile (DV/RTS MH/MH).
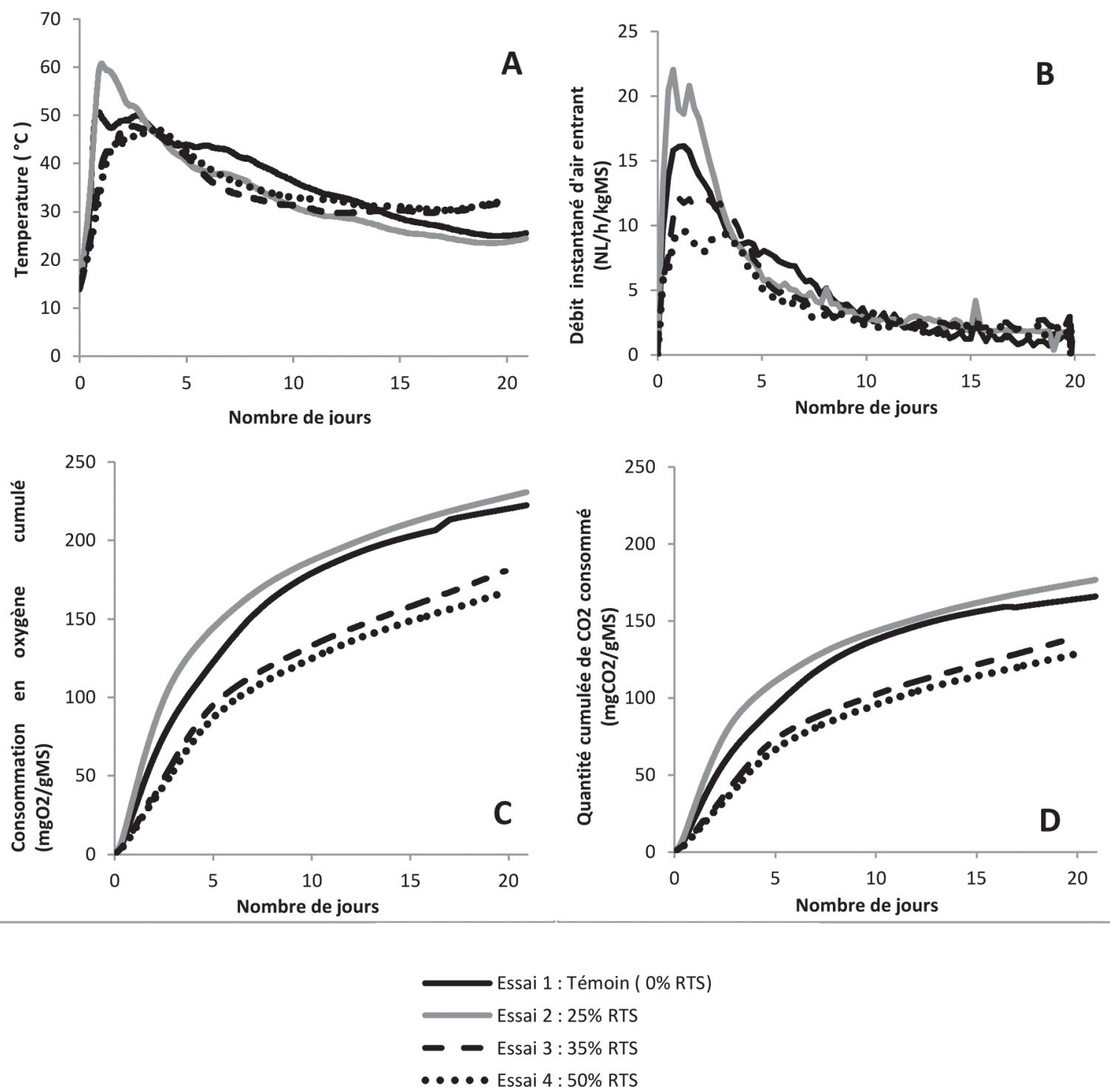

3.2. Etude du comportement en cocompostage des résidus de toilettes sèches et déchets verts

Les suivis de la température, production de $\mathrm{CO}_{2}$, consommation d' $\mathrm{O}_{2}$ et débit d'air, au cours des essais de compostage réalisés en pilote sur 21 jours, sont présentés dans la Figure 2 $\left(25,35\right.$ et $50 \%_{M H}$ RTS ; et témoin 100\% de DV).

Les résultats montrent que plus la proportion de DV augmente dans le mélange (RTS et DV), plus la réactivité biologique des matrices est importante. Les courbes de consommation en $\mathrm{O}_{2}$ et de production de $\mathrm{CO}_{2}$, similaires en termes de cinétique, mettent en évidence des comportements similaires entre les essais I (Témoin) et 2 (25\% ${ }_{M H}$ RTS) d'une part, et les essais 3 $\left(35 \%_{M H}\right.$ RTS) et $4\left(50 \%_{M H}\right.$ RTS ) d'autre part. En présence de
25\% de RTS, l'activité biologique est pratiquement similaire à celle observée sur l'essai témoin $100 \%$ DV. Cette observation tend à indiquer que la présence de 25\% de RTS n'a pas de conséquence significative sur le comportement biologique en conditions de compostage. En outre, la présence de 25\% de RTS se traduit par un bénéfice d'augmentation de la température au démarrage de l'aération, suivi par un léger déficit de température au-delà de 5 jours de compostage. Les essais 3 et 4 réalisés à respectivement 35 et $50 \%_{M H}$ RTS conduisent à obtenir des courbes de température, de consommation d' $\mathrm{O}_{2}$ et de production de $\mathrm{CO}_{2}$ pratiquement identiques, correspondant à une activité respiratoire globalement plus faible que celle observée sur l'essai témoin et l'essai à $25 \%_{M H}$ RTS. La température maximale atteinte est inférieure à $50^{\circ} \mathrm{C}$ et les débits de renouvellement d'air, associé au besoin d'injection d'air pour maintenir une teneur en $\mathrm{O}_{2}$ de 15\% dans le composteur, sont significativement plus faibles (cf.Tableau 2). 
Par ailleurs, les taux de conversion de la matière organique calculés sur la base de la production de $\mathrm{CO}_{2}$ (cf. Tableau 5) indiquent sa plus forte biodégradation sur l'essai I témoin et l'essai 2 à $25 \%_{M H}$ RTS, de valeurs respectives I5, I \% et 15,7\%, contre $11,5 \%$ et $10,6 \%$ pour les essais 3 et 4 à 35 et $50 \%{ }_{\mathrm{MH}}$ RTS, respectivement. Le mélange $25 \%_{M H}$ RTS $-75 \%_{M H}$ DV présente donc un taux de conversion de la matière organique équivalent à l'essai témoin 100\% DV, avec en outre un léger gain sur la vitesse de bioconversion (augmentation de la constante cinétique k de ler ordre, (cf. Tableau 5).

A l'issue des quatre essais de compostage réalisés, nous retenons que l'activité biologique (température, constante cinétique et taux de conversion) tend globalement à baisser en présence de RTS au-delà de $25 \%$. La baisse d'activité en présence de 35 et $50 \%$ de RTS peut s'expliquer par deux hypothèses. La première hypothèse est de considérer que la matière organique présente dans les RTS serait moins biodégradable que celle contenue dans les DV. C'est l'hypothèse la plus plausible au regard des résultats obtenus au cours du test $\mathrm{ARS}_{14}$ de respirométrie (cf. Tableau 2). Exprimée en tenant compte de la teneur en matière volatile, l'activité respiratoire aérobie des RTS est de l'ordre de $110 \mathrm{mg} \mathrm{O}_{2} \cdot \mathrm{g}_{\mathrm{MV}}^{-1}$ et de 210 $m g{ }_{02} \cdot g^{-1}$ MV pour les DV initiaux. Cette différence significative de biodégradabilité s'explique aisément par la nature lignocellulosique des RTS solides, constitués majoritairement de sciure et copeaux de bois. La seconde hypothèse est de considérer l'influence de la structure particulaire des RTS dont l'influence sur le compostage est double : effet sur l'aération de la matière et sur la rétention d'humidité. Dans notre cas de figure, nous remarquons qu'une teneur en eau initiale de 68,3\% permet à la matrice composée de $75 \%$ de déchet vert et $25 \%$ de résidus de toilettes sèches d'obtenir une meilleure activité biologique et taux de conversion de la matière organique que la matrice composée de $100 \%$ de déchets verts initialement constituée de $61 \%$ d'humidité et $10 \%$ plus riche en matière organique que la matrice d'essai 75\% DV. De même, les matrices d'essais 65\% DV et 50\% DV avec respectivement un taux d'humidité initiale des $70,6 \%$ et $73,4 \%$ fournissent des activités biolo- giques et taux de conversion nettement inférieurs aux deux autres essais précédents. Ces informations nous permettent de montrer que la teneur en eau est un facteur influençant l'activité biologique et le taux de conversion de la matière organique.

Des milieux trop humides freinent la dégradation de la matière organique et la production de $\mathrm{CO}_{2}$. En effet, le transfert d'oxygène (qui contrôle l'activité biologique aérobie) au cours d'un processus de biodégradation est conditionné par l'humidité : si le compost est trop humide, le transfert de l'oxygène est ralenti, ce qui diminue la production de $\mathrm{CO}_{2}$ (Yulipriyanto, 200I). La Figure 3 montre les valeurs de teneur en humidité mesurées en fin d'incubation pour les différents essais. Pour chaque matrice, des quantités de $10 \mathrm{~kg}$ ont été prélevées (en 5 couches) sur la colonne du déchet de haut en bas afin de mesurer la teneur moyenne en eau dans chaque couche.

Nous constatons alors que les matrices d'études 65\% DV et 50\% DV sont caractérisées par des teneurs en eau importantes au niveau du compartiment inférieur des réacteurs. En effet, des quantités de lixiviats assez importantes (6,6 et 4,4 litres) ont été collectées en fin d'incubation dans chacun de ces deux réacteurs. Les réacteurs sont alimentés en air de bas en haut. Ces valeurs importantes de teneur en eau dans les couches inférieures des réacteurs ont éventuellement influencé la diffusion de l'air dans toute la matrice (chemins préférentiels).

\subsection{Caractérisation des résidus après compostage}

\section{Analyse globale}

Les résultats regroupés dans le Tableau 6 indiquent une baisse de la DCO et du rapport C/N après 3 semaines d'incubation. Les plus faibles valeurs d'azote organique sont observées dans les essais à fortes teneurs en RTS. Les valeurs de $\mathrm{pH}$ ont légèrement augmenté pour les matrices 100\% DV et 25\% RTS. Cette augmentation du $\mathrm{pH}$ peut être imputée à la dégradation des acides gras à chaînes courtes et à la libération d'ammoniac

Tableau 5. Données de suivi des essais pilotes de compostage

\begin{tabular}{|c|c|c|c|c|c|c|}
\hline Essai & DV/RTS & $\mathrm{T}_{\max }\left({ }^{\circ} \mathrm{C}\right)$ & $\begin{array}{l}\text { Cons. d'oxygène } \\
\left(\mathrm{mg} \mathrm{O}_{2} \cdot \mathrm{g}^{-1} \mathrm{MS}\right)\end{array}$ & $\begin{array}{l}\text { Prod. de } \mathrm{CO}_{2}\left(Q_{\mathrm{CO}_{2}}\right) \\
\quad\left(\mathrm{mgCO} \mathrm{CO}_{2} \cdot \mathrm{g}_{\mathrm{MS}}\right)\end{array}$ & $\begin{array}{c}\mathbf{k} \\
\left(\mathbf{j}^{-1}\right)\end{array}$ & $\begin{array}{c}\text { Taux de } \\
\text { conversion } \\
\text { de la MO } \\
\tau\left(\%_{\mathrm{MV}}\right)\end{array}$ \\
\hline EI & 100 & 50,7 & 222 & 166 & 0,17 & 15,07 \\
\hline E2 & $75 / 25$ & 60,8 & 230 & 176 & 0,19 & 15,75 \\
\hline E3 & $65 / 35$ & 47,8 & 181 & 139 & 0,15 & 11,53 \\
\hline E4 & $50 / 50$ & 47,1 & 167 & 128 & 0,13 & 10,63 \\
\hline
\end{tabular}

k= Constante de cinétique exprimée par rapport à la quantité de MS dans le réacteur (équation de cinétique d'ordre I)

$\tau=$ taux de conversion de la matière organique exprimé en pourcentage de matière organique converti en $\mathrm{CO}_{2}$ ( $c f$. point 2.2 équation 2 ); $\mathrm{QCO}$, la production cumulée de $\mathrm{CO}_{2}$ et le taux de matière volatile dans la matière sèche présente dans le réacteur. 
Figure 3 : Répartition de l'humidité au sein des matrices en fin de cycle de compostage en pilote (fermentation chaude de 21 jours).

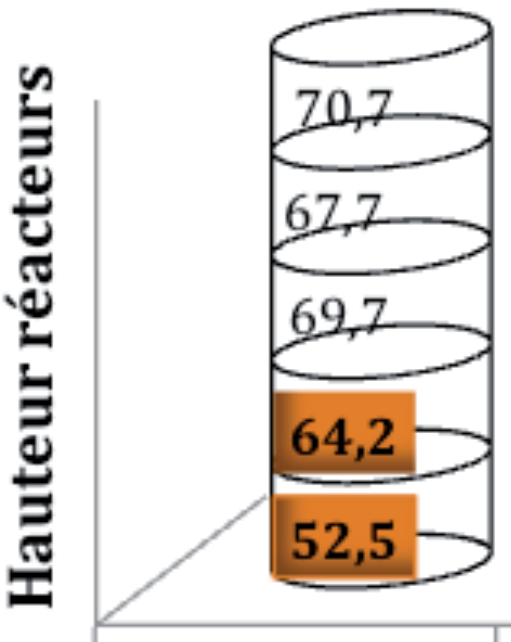

0\%RTS $100 \%$ DV

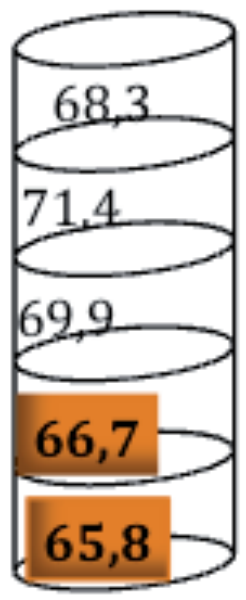

25\% RTS

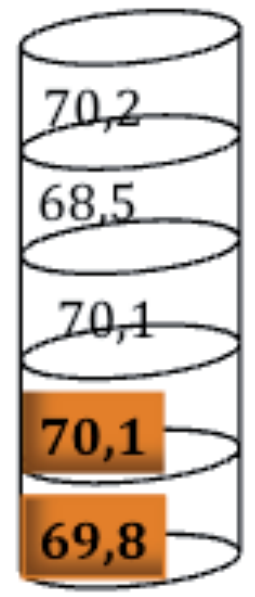

$35 \%$ RTS

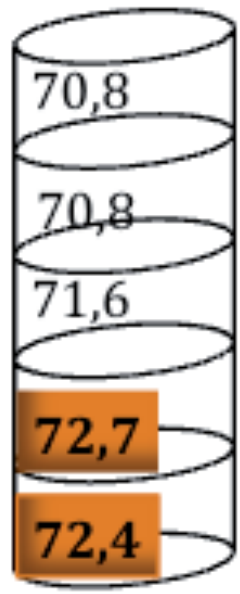

50\% RTS dans le processus d'ammonification suite à la dégradation des acides organiques dans le compost.

Les analyses de DCO $\left(900\right.$ - $\left.1050 \mathrm{mg} \cdot \mathrm{g}^{-1}\right), \mathrm{C} / \mathrm{N}(23-34)$ et $\mathrm{NO}_{3}-\mathrm{NH}_{3}(<\mathrm{I})$ montrent que le déchet, après 3 semaines d'incubation, n'est dans aucun des essais un compost stabilisé (Mustin, 1987 ; Forster et al., 1993 ; Iglesias-Jimenez \& PerezGarcia, 1989). Mustin souligne que la maturité d'un compost est jugée atteinte lorsque la DCO est inférieure à $350 \mathrm{mg} \cdot \mathrm{g}^{-1}$ De même, les autres auteurs indiquent que des rapports $\mathrm{C} / \mathrm{N}$ inférieurs à 20 et un rapport $\mathrm{NO}_{3}-\mathrm{NH}_{4}$ supérieur à I sont caractéristiques de composts mûrs.
Devenir des micropolluants pharmaceutiques et stupéfiants

Le Tableau 7 résume les résultats d'analyse des molécules pharmaceutiques (Tableau 7.A), molécules médicamenteuses détournées de leur usage, et des stupéfiants (Tableau 7.B) obtenus dans les matrices d'études après les 3 semaines d'incubation, ainsi que les taux d'abattement de ces micropolluants ciblés. Pour rappel, l'analyse par screening de la matrice initiale 100\% RTS a ciblé 12 molécules pharmaceutiques parmi lesquelles trois molécules n'ont pas été détectées (carbamazé-

Tableau 6 : Caractéristiques globales des résidus après compostage (fermentation chaude de 21 jours).

\begin{tabular}{|c|c|c|c|c|c|c|c|c|c|}
\hline $\begin{array}{c}\# \\
\text { Essai }\end{array}$ & DV/RTS & $\mathrm{pH}$ & $\begin{array}{c}\text { MS } \\
\left(\%_{M H}\right)\end{array}$ & $\begin{array}{c}\text { MV } \\
\left(\%_{M S}\right)\end{array}$ & $\begin{array}{c}\text { DCO } \\
\left(\mathrm{g} / \mathrm{kg}_{\mathrm{MS}}\right)\end{array}$ & $\begin{array}{c}\text { N-NT } \\
\left(\mathrm{g} / \mathrm{kg}_{\mathrm{MS}}\right)\end{array}$ & $\begin{array}{l}\mathrm{N}-\mathrm{NH}_{3} \\
\left(\mathrm{~g} / \mathrm{kg}_{\mathrm{MS}}\right)\end{array}$ & $\begin{array}{c}\mathbf{N}-\mathbf{N}_{\text {org }} \\
\left(\mathrm{g} / \mathrm{kg}_{\mathrm{MS}}\right)\end{array}$ & $\mathbf{C} * \mathbf{N}$ \\
\hline El & 100 & 9,2 & 34,9 & 76,0 & 998 & 16,2 & $<1,2$ & 15,0 & 23,1 \\
\hline E2 & $75 / 25$ & 9,1 & 31,5 & 76,4 & 1070 & $15, \mid$ & $<0,9$ & 14,3 & 24,5 \\
\hline E3 & $65 / 35$ & 8,7 & 31,0 & 74,7 & 1095 & 13,6 & $<1,0$ & 12,6 & 26,6 \\
\hline E4 & $50 / 50$ & 8,5 & 28,4 & 77,8 & 1130 & 12,1 & $<1,0$ & $|1|$, & 34,4 \\
\hline
\end{tabular}

\footnotetext{
* C déterminé par calcination
} 
Tableau 7. Analyse des micropolluants pharmaceutiques $(A)$ et des stupéfiants $(B)$ des résidus après compostage (fermentation chaude de 21 jours)

\begin{tabular}{|c|c|c|c|c|}
\hline \multirow{2}{*}{ A } & \multirow[b]{2}{*}{ Molécule } & \multicolumn{3}{|c|}{$\begin{array}{l}\text { Concentration (ng/g } \mathrm{g}_{\text {MS }} \text { ) et } \\
\text { Taux d'abattement }(\%)\end{array}$} \\
\hline & & $\begin{array}{l}\text { DV/RTS } \\
75 / 25\end{array}$ & $65 / 35$ & $50 / 50$ \\
\hline Antalgiques & Paracétamol & $\begin{array}{l}\text { ND } \\
(100)\end{array}$ & $\begin{array}{c}102 \\
(-)\end{array}$ & $\begin{array}{l}\text { ND } \\
(100)\end{array}$ \\
\hline \multirow{4}{*}{$\begin{array}{l}\text { anti-inflammatoire } \\
\text { non stéroïdiens }\end{array}$} & Ac. Salicylique & $\begin{array}{c}174 \\
(-)\end{array}$ & $\begin{array}{c}245 \\
(-)\end{array}$ & $\begin{array}{l}196 \\
(9)\end{array}$ \\
\hline & Diclofénac & $\begin{array}{l}\text { ND } \\
(100)\end{array}$ & $\begin{array}{l}\text { ND } \\
(100)\end{array}$ & $\begin{array}{l}\text { ND } \\
(100)\end{array}$ \\
\hline & lbuprofène & $\begin{array}{l}30 \\
(75)\end{array}$ & $\begin{array}{l}29 \\
(83)\end{array}$ & $\begin{array}{c}29 \\
(89)\end{array}$ \\
\hline & Kétoprofène & $\begin{array}{l}\text { ND } \\
(100)\end{array}$ & $\begin{array}{l}\text { ND } \\
(100)\end{array}$ & $\begin{array}{l}\text { ND } \\
(100)\end{array}$ \\
\hline \multirow[t]{2}{*}{ Bêtabloquants } & Aténolol & $\begin{array}{c}\text { ND } \\
\text { Non estimé }\end{array}$ & $\begin{array}{l}\text { ND } \\
\text { Non estimé }\end{array}$ & $\begin{array}{c}\text { ND } \\
\text { Non estimé }\end{array}$ \\
\hline & Propanolol & $\begin{array}{l}\text { ND } \\
(100)\end{array}$ & $\begin{array}{l}N Q \\
(\sim 100)\end{array}$ & $\begin{array}{l}\mathrm{NQ} \\
(\sim 100)\end{array}$ \\
\hline \multirow[t]{2}{*}{ Hormones } & Ethynil oestradiol & $\begin{array}{l}\text { ND } \\
\text { Non estimé }\end{array}$ & $\begin{array}{l}\text { ND } \\
\text { Non estimé }\end{array}$ & $\begin{array}{l}\text { ND } \\
\text { Non estimé }\end{array}$ \\
\hline & Ciprofloxacine & $\begin{array}{l}\text { ND } \\
(100)\end{array}$ & $\begin{array}{l}\text { ND } \\
(100)\end{array}$ & $\begin{array}{l}\text { ND } \\
(100)\end{array}$ \\
\hline \multirow[t]{2}{*}{ Antibiotiques } & Econazole & $\begin{array}{c}\mathrm{NQ} \\
(\sim 100)\end{array}$ & $\begin{array}{c}N Q \\
(\sim 100)\end{array}$ & $\begin{array}{c}5 \\
(82)\end{array}$ \\
\hline & Erythromycine & $\begin{array}{l}21 \\
(-)\end{array}$ & $\begin{array}{l}21 \\
(-)\end{array}$ & $\begin{array}{l}21 \\
(-)\end{array}$ \\
\hline Antidépresseurs & Carbamazépine & $\begin{array}{c}\text { ND } \\
\text { Non estimé }\end{array}$ & $\begin{array}{c}\text { ND } \\
\text { Non estimé }\end{array}$ & $\begin{array}{c}\text { ND } \\
\text { Non estimé }\end{array}$ \\
\hline
\end{tabular}

(ND) non détecté - (NQ) non quantifié (-) : valeurs négatives

pine, l'aténolol, et éthynil œstradiol). A l'issue de la période d'incubation, sur les 9 autres molécules restantes ayant été soumises aux 3 essais en présence de RTS (25\%, 35\% et 50\%RTS), nous retenons que :

- 3 molécules sont non détectées dans les matrices finales des 3 matrices étudiées (abattement $=100 \%$ ) ; c'est le cas du diclofénac, du kétoprofène et de la ciprofloxacine ;

- 4 autres molécules ont globalement de bonnes valeurs d'abattement comprises entre [75 et 100], présentant ainsi des valeurs finales de concentration de l'ordre de faibles valeurs de concentration finales mesurées $\left(<30 \mathrm{ng} \cdot \mathrm{g}^{-1}\right.$ ms $)$, à des valeurs non détectées : c'est le cas du paracétamol, propanolol, éconazole et ibuprofène. Cependant, soulignons qu'il n'y a pas eu de dégradation du paracétamol dans l'essai à 35\% RTS (abattement négatif).

- 2 autres molécules ont globalement des concentrations finales supérieures aux concentrations initiales (abattement $<0$ ), reflétant ainsi la non dégradation ou persistance de celles-ci au cours de la période d'incubation ; c'est le cas de l'aspirine et l'érythromicine. En effet la réduction de la masse des matrices d'étude lors de leur biodégradation et la persistance des molécules dans celles-ci pourraient expliquer les valeurs de concentrations finales supérieures aux concentrations initiales.
L'analyse des stupéfiants dans la matrice initiale 100\%RTS (sèche) a ciblé II molécules, parmi lesquelles 7 ne sont pas détectées initialement sur échantillon solide ; c'est le cas de la 6-monoacétylmorphine, de la benzoylecgonine et de l'ecgonine méthylester (métabolites de la cocaïne), de la méthamphétamine, du $\mathrm{THC}$, du II-OH-THC et du $\mathrm{THC}-\mathrm{COOH}$. Sur les 4 molécules restantes ayant été soumises aux essais (25\%, 35\% et 50\% RTS), nous retenons que 3 molécules ont un abattement $>65 \%$ (morphine, MDMA, ecstasy, amphétamine) et que la MDA a un abattement non stable (100-32\% et valeur négative). La diminution nette des concentrations de MDMA est due à l'effet de dilution et très certainement à la dégradation ou biotransformation menant à l'augmentation du produit actif MDA.

En outre, une analyse qualitative a été effectuée sur chaque échantillon issu des essais de compostage. II a été observé que la kétamine, la norkétamine et le tramadol sont toujours présents dans les échantillons.

Les teneurs sont de l'ordre de la dizaine de $\mu g \cdot g^{-1}$ à quelques ng. $g^{-1}$ pour les molécules étudiées, soit des valeurs très faibles, d'autant plus que les techniques analytiques employées pour 
Suite : Tableau 7. Analyse des micropolluants pharmaceutiques $(A)$ et des stupéfiants $(B)$ des résidus après compostage (fermentation chaude de 21 jours)

\begin{tabular}{|c|c|c|c|c|}
\hline \multirow{2}{*}{ B } & \multirow[b]{2}{*}{ Molécule } & \multicolumn{3}{|c|}{$\begin{array}{c}\text { Concentration }\left(\mathrm{ng} / \mathrm{g}_{\mathrm{MS}}\right) \text { et } \\
\text { Taux d'abattement }\end{array}$} \\
\hline & & $\begin{array}{l}\text { DV/RTS } \\
\mathbf{7 5 / 2 5}\end{array}$ & $65 / 35$ & $50 / 50$ \\
\hline \multirow[t]{6}{*}{ Molécules médicamenteuses détournées de leur usage } & $\begin{array}{l}\text { Kétamine } \\
\text { Norkétamine } \\
\text { Tramadol }\end{array}$ & $\begin{array}{c}+ \\
(0) \\
+ \\
(0) \\
+ \\
(0)\end{array}$ & $\begin{array}{c}+ \\
(0) \\
+ \\
(0) \\
+ \\
(0)\end{array}$ & $\begin{array}{c}+ \\
(0) \\
+ \\
(0) \\
+ \\
(0)\end{array}$ \\
\hline & $\begin{array}{l}\text { Morphine } \\
\text { 6MAM }\end{array}$ & $\begin{array}{l}\text { ND } \\
(100) \\
\text { ND } \\
\text { Non } \\
\text { estimé }\end{array}$ & $\begin{array}{c}\text { ND } \\
(100) \\
\text { ND } \\
\text { Non estimé }\end{array}$ & $\begin{array}{c}\text { ND } \\
(100) \\
\text { ND } \\
\text { Non estimé }\end{array}$ \\
\hline & Amphétamine & $\begin{array}{l}\text { ND } \\
(100)\end{array}$ & $\begin{array}{l}12,5 \\
(68)\end{array}$ & $\begin{array}{l}\text { ND } \\
(100)\end{array}$ \\
\hline & Méthamphétamine & $\begin{array}{l}\text { ND } \\
\text { Non } \\
\text { estimé }\end{array}$ & $\begin{array}{l}3,4 \\
(-)\end{array}$ & $\begin{array}{l}\text { ND } \\
\text { Non estimé }\end{array}$ \\
\hline & MDMA (Ecstasy) & $\begin{array}{l}442 \\
(80)\end{array}$ & $\begin{array}{l}132 \\
(96)\end{array}$ & $\begin{array}{l}378 \\
(92)\end{array}$ \\
\hline & MDA & $\begin{array}{l}\text { ND } \\
(100)\end{array}$ & $\begin{array}{l}193 \\
(32)\end{array}$ & $\begin{array}{l}1190 \\
(-)\end{array}$ \\
\hline & $\mathrm{THC}$ & $\begin{array}{c}\text { ND } \\
\text { Non } \\
\text { estimé }\end{array}$ & $\begin{array}{l}\text { ND } \\
\text { Non estimé }\end{array}$ & $\begin{array}{c}\text { ND } \\
\text { Non estimé }\end{array}$ \\
\hline Cannabinoïdes & $\begin{array}{l}\text { I I-OH-THC } \\
\text { THC-COOH }\end{array}$ & $\begin{array}{c}\text { ND } \\
\text { Non } \\
\text { estimé } \\
+ \\
(-)\end{array}$ & $\begin{array}{l}\text { ND } \\
\text { Non estimé } \\
58 \\
(-)\end{array}$ & $\begin{array}{c}\text { ND } \\
\text { Non estimé } \\
+ \\
(-)\end{array}$ \\
\hline
\end{tabular}

6MAM : 6-monoacétylmorphine (marqueur de l'héroine) ;

MDMA : 3,4-méthylène-dioxy-methamphétamine ;

MDA : 3,4 méthylène-dioxy-amphétamine (dérivé et métabolite de la MDMA);

THC : tétra-hydro-cannabinol ;

I I-OH-THC : I I-hydroxy-tétrahydrocannabinol (métabolite du THC)

THC-COOH : carboxy-tétra-cannabinol (métabolite duTHC)

ND : Non Détecté.- + : présence

l'extraction et l'analyse ont été développées au départ pour d'autres types de milieux que ceux analysés ici. De plus, les faibles concentrations de ces molécules et la présence de déchets verts dans ces matrices d'étude, augmentent l'apport en interférents conduisant à limiter la quantification réelle des MPP et stupéfiants. Des travaux sont en cours dans l'objectif de trouver les meilleures méthodes analytiques permettant de mieux quantifier ces molécules dans les matrices solides.

\section{CONCLUSION}

Cette étude a permis d'évaluer le comportement des résidus de toilettes sèches sous des conditions de co-compostage en présence de déchets verts. Quatre proportions de mélange ont été testées sur une période d'incubation de 3 semaines (0\%, 25\%, 35\%, 50\% RTS) dans des conditions régulées 
d'aération. Les résultats montrent que plus la proportion de RTS augmente, plus la température, la consommation en $\mathrm{O}_{2}$ et la production de $\mathrm{CO}_{2}$ diminuent. Le fort taux d'humidité et la plus faible biodégradabilité de la matière organique contenue dans les résidus de toilettes sèches en sont les principales causes. Cependant, pour 25\% de RTS, le taux de conversion de la matière organique est comparable à celui déterminé sur les déchets verts sans ajout de RTS (I00\% DV) après 3 semaines d'incubation. La bonne réactivité du mélange RTS-DV 25-75 est encourageante pour envisager le co-compostage de RTS sur des plateformes de compostage de déchets verts. L'analyse des micropolluants pharmaceutiques, stupéfiants et xénobiotiques avant et après compostage a permis de mettre en évidence des réductions de concentration des molécules pharmaceutiques telles que le diclofénac, le kétoprofène, la ciprofloxacine, le paracétamol, le propanolol, l'éconazole et l'ibuprofène et également de certains stupéfiants tels la MDMA, l'amphétamine et la morphine. En revanche, l'aspirine, l'érythromicine, la kétamine, la norkétamine, le tramadol et la MDA ont montré des caractères de persistance dans les matrices étudiées à l'issue des 3 semaines de co-compostage. Ces résultats ne sont pas suffisants pour conclure de manière générale sur l'innocuité chimique du compost issu du traitement. Des périodes d'incubation plus longues permettraient de fournir des informations plus complètes sur le comportement de ces molécules en tenant compte de la phase de maturation. Enfin, le suivi des agents pathogènes après le cycle de fermentation chaude n'a pas fait l'objet d'étude ici, compte tenu de leur faible concentration (inférieure au seuil d'innocuité) lors de la caractérisation initiale des RTS après six mois de stockage. Néanmoins, l'innocuité biologique devra nécessairement être prise en considération avant d'envisager d'intégrer des RTS dans une filière de compostage de déchets verts, ainsi que d'autres paramètres tels que la perception des risques par les utilisateurs.

\section{Références bibliographiques}

AFNOR NF U 44-095 (2002). Amendements organiques - Composts contenant des matières drintérêt agronomique, issues du traitement des eaux

AFNOR NF EN I5- 169 (2005). Caractérisation des déchets - Détermination de la perte au feu des déchets, des boues et des sédiments. Mesure de la teneur en résidu calciné par calcination à $550^{\circ} \mathrm{C}$.

Albrecht R. (2007). CO-compostage de boues de station d'épuration et de déchets verts : nouvelle méthodologie du suivi des transformations de la matière organique. [en ligne]. Thèse de l'Université Paul CEZANNE, 2007,189 p.

Bacheley H., Francou C., Chevallier M., \& Poitrenaud M. (2008). Control of gas emissions during composting of municipal solid waste, municipal Biowaste and industrial biowaste. ORBIT 2008.

Baumeyer A. (2003). New Toilets for Indian Slums: Nutrients Mass Balance of a Co-Composting Plant in Bangalore, India [en ligne]. Thèse de l'Université de HOCHSCHULE WADENSWIL, Allemagne, 2003, 100 p. Disponible sur http://www2.gtz.de/Dokumente/oe44/ecosan/en-nutrient-mass-balance-cocomposting-slum-bangalore-india-2003.pdf. (consulté le 0।.09. 20।4)

Brinton W. F., Evans E., Droffner M. L. \& Brinton R. B. (1995). Standardized test for evaluation of compost self-heating. BioCycle. 64-69.

Daughton C.D. \& Ternes, T.A. (1999). Pharmaceuticals and personal care products in the environment: agents of subtle change? Environ Health Perspect. I07 Suppl 6: pp : 907-38.

Chenxi W., Spongberg A.L. \& Witter J.D. (2008). Determination of the persistence of pharmaceuticals in biosolids using liquid-chromatography tandem mass spectrometry. Chemosphere, 7I (4) pp. 5 I3-5 I8. doi: I0.10 I6/j.chemosphere.2008.06.026

Coetsier C. (2009). Approche intégrée de la gestion environnementale des produits pharmaceutiques dans des rejets de stations d'épuration urbaines et leur milieu récepteur: Occurrence, impact et traitements tertiaires d'élimination [en ligne]. Thèse de I'Université Montpellier II, 2009, 293 p.

De Guardia A., Mallard P., Teglia C., et al, (2010). Comparison of five organic wastes regarding their behavior during composting, Part2: nitrogen dynamic. Waste Management, 30 (3), pp : 4I5-425.

Elain C. (2007) Un petit coin pour soulager la planète. Edition Eauphilane. 5 rue d'Avesnières 53000 Laval, 2007, 288 pages. ISBN 978 - 2- $9530877-0$ - 3.

Forster, J. C., W. Zech, and E.Würdinger. (1993). Comparison of chemical and microbial methods for the characterization of the maturity of composts from contrasting sources. Biol. Fertil. Soils, 16:93-99.

Francou C. (2003). Stabilisation de la matière organique au cours du compostage de déchets urbains : influence de la nature des déchets et du procédé de compostage - Recherche d'indicateurs pertinents [en ligne], Thèse de Doctorat d'Agronomie, Institut National Agronomique Paris-Grignon, 2003, $289 \mathrm{p}$

Habart J., Tlustoš P., Han A., et al. (2010). The role of aeration intensity, temperature regimes and composting mixture on gaseous emission during composting. Compost Science \& Utilization pages 194-200, Volume I8, Issue 3.

Iglesias-Jimenez, E. \& Perez-Garcia.V. (1989). Evaluation of city refuse compost maturity: A review. Biological Wastes, 27, pp : I I5- 142.

Jenkins J. (2005). Humanure handbook : a guide to composting Human Manure. Edition Jenkins Publishing. PO Box 428, Grove city, PA I6I27 USA, 255 pages. ISBN - I $0: 0$ - 9644258-3-I.

Jjemba P.K. (2006). Excretion and ecotoxicity of pharmaceutical and personal care products inthe environment. Ecotoxicology and Environmental Safety, 63(I), pp : I 13-130.

Jones O.A., Lester, J. N. \& Voulvoulis, N. (2005). Pharmaceuticals: a threat to drinking water? Trends in Biotechnology, 23 (4), pp : $163-167$.

ISO EN NF 14235 (1998). Soil quality - Determination of organic carbon by sulfochromic oxidation.

Kümmerer K. (200 I). Drugs in the environment: emission of drugs, diagnostic aids and disinfectants into wastewater by hospitals in relation to other sources - a review. Chemosphere, 45 (6-7), pp.. 957-969.

Mustin M. (1987). Le compost: Gestion de la matière organique. Editions François DUBUS. 35, rue Marthurin-Régnier 750I5 PARIS, 1987, 954 pages. ISBN 2-864-72008-6.

ODCE Library (20l4). http://www.oecd-ilibrary.org/fr/environment/lignesdirectrices-de-l-ocde-pour-les-essais-de-produits-chimiques-section-3-degradation-et-accumulation_20745834.

Pynnönen S. \& Tuhkanen T. (20I2). Environmental impact of micropollutants present in urine. Dry Toilet Conference 2012, Book of Abstracts, 4th International Dry Toilet Conference, 22-24 August 2012, Tampere, Finland. 
Soulier C.,Gabet V., Lardy S. et al., (201 I). Zoom sur les substances pharmaceutiques : présence, partition, devenir en station d'épuration. Extrait numérique de Techniques Sciences Méthodes $n^{\circ}$ I/2 de 20II. Disponible sur : https://projetamperes.cemagref.fr/illustrations/63-77-SOULIER.pdf (consulté le 01.09 .2014$)$

Winker M. (2009). Pharmaceutical residues in urine and potential risks related to usage as fertilizer in agriculture [en ligne].Thèse de l'Université technique de Hambourg harburg, 2009, I I 8 p. Disponible sur : http://doku.b.tu-harburg. de/volltexte/2009/557/pdf/PhD_Thesis_Winker.pdf (consulté le 01.09. 2014)

Yulipriyanto H. (200I). Emission d'effluents gazeux lors du compostage de substrats organiques en relation avec l'activité microbiologique (nitrification/ dénitrification) [en ligne].Thèse de l'Université de Rennes I, 200 I, 210 p. Disponible sur http://tel.archives-ouvertes.fr/docs/00/65/47/0I/PDF/These_Hieronymus_Yulipriyanto_200I.pdf (consulté le 01.09.2014)

Zuccato E., Calamari D., Natangelo, M. \& Fanelli R. (2000). Presence of therapeutic drugs in the environment. The Lancet, 355 (92 I7), p.p : I 789- 1790.

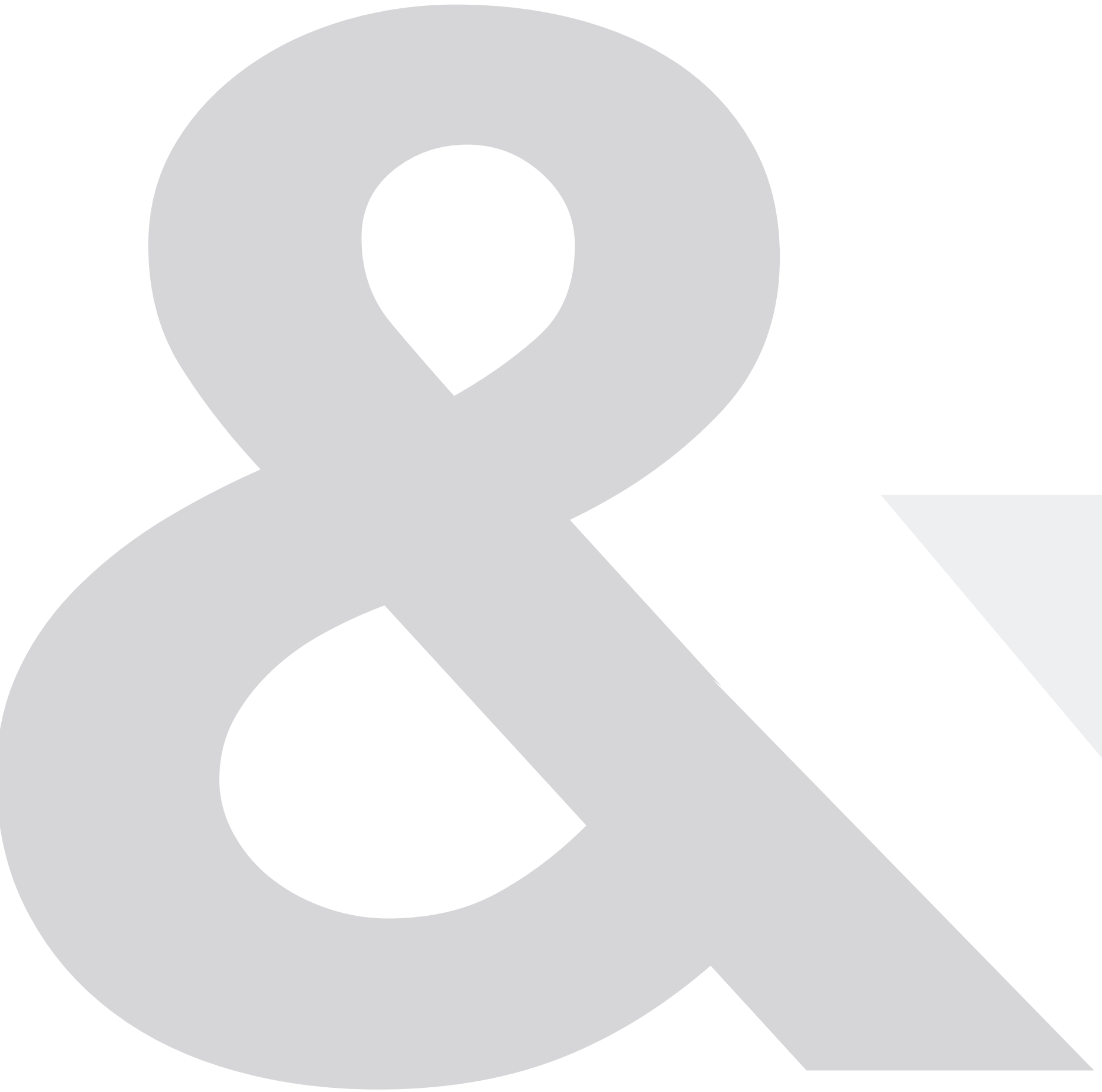

